\title{
A Non-ergodic Spectral Acceleration Ground Motion Model for California Developed with Random Vibration Theory
}

Grigorios Lavrentiadis ( $\sim$ glavrent@berkeley.edu )

UC Berkeley: University of California Berkeley https://orcid.org/0000-0001-6546-1340

Norman A. Abrahamson

UC Berkeley: University of California Berkeley

\section{Research Article}

Keywords: Probabilistic seismic hazard analysis , Ground-motion model , Random vibration theory

Posted Date: July 22nd, 2021

DOI: https://doi.org/10.21203/rs.3.rs-712861/v1

License: (c) (1) This work is licensed under a Creative Commons Attribution 4.0 International License.

Read Full License 


\title{
A Non-ergodic Spectral Acceleration Ground Motion Model for California Developed with Random Vibration Theory
}

\author{
Grigorios Lavrentiadis • Norman A. Abrahamson
}

Received: date / Accepted: date

\begin{abstract}
A new approach for creating a non-ergodic $P S A$ ground-motion model $(\mathrm{GMM})$ is presented which account for the magnitude dependence of the non-ergodic effects. In this approach, the average $P S A$ scaling is controlled by an ergodic $P S A$ GMM, and the non-ergodic effects are captured with non-ergodic $P S A$ factors, which are the adjustment that needs to be applied to an ergodic PSA GMM to incorporate the non-ergodic effects. The non-ergodic $P S A$ factors are based on $E A S$ non-ergodic effects and are converted to $P S A$ through Random Vibration Theory (RVT). The advantage of this approach is that it better captures the non-ergodic source, path, and site effects through the small magnitude earthquakes. Due to the linear properties of Fourier Transform, the EAS non-ergodic effects of the small events can be applied directly to the large magnitude events. This is not the case for $P S A$, as response spectrum is controlled by a range of frequencies, making $P S A$ non-ergodic effects depended on the spectral shape which is magnitude dependent.

Two PSA non-ergodic GMMs are derived using the ASK14 (Abrahamson et al. 2014) and CY14 (Chiou and Youngs, 2014) GMMs as backbone models, respectively. The non-ergodic EAS effects are estimated with the LAK21 (Lavrentiadis et al. In press) GMM. The RVT calculations are performed with the V75 (Vanmarcke, 1975) peak factor model, the $D_{a 0.05-0.85}$ estimate of AS96 (Abrahamson and Silva, 1996) for the ground-motion duration, and BT15 (Boore and Thompson. 2015) oscillator-duration model. The California subset of the NGAWest2 database (Ancheta et al. 2014) is used for both models.

The total aleatory standard deviation of the two non-ergodic PSA GMMs is approximately 30 to $35 \%$ smaller than the total aleatory standard deviation of the
\end{abstract}

Grigorios Lavrentiadis

Department of Civil Engineering

University of California, Berkeley

Berkeley, California 94720 U.S.A.

E-mail: glavrent@berkeley.edu

Norman A. Abrahamson

Department of Civil Engineering

University of California, Berkeley

Berkeley, California 94720 U.S.A.

E-mail: abrahamson@berkeley.edu 
corresponding ergodic PSA GMMs. This reduction has a significant impact on hazard calculations at large return periods. In remote areas, far from stations and past events, the reduction of aleatory variability is accompanied by an increase of epistemic uncertainty.

Keywords Probabilistic seismic hazard analysis · Ground-motion model · Random vibration theory

\section{Introduction}

Ground-motion models (GMMs) are used to estimate the distribution of a groundmotion intensity measure $(I M)$ for a given earthquake scenario. The most common IM is pseudo-spectral acceleration $(P S A)$ as it is a good estimator of seismic loading for a wide range of structures. $P S A$ is defined as the absolute maximum response of a single-degree-of-freedom oscillator (SDOF) to an input ground motion. SDOFs are defined by their natural period $\left(T_{0}\right)$ or natural frequency $\left(f_{0}=1 / T_{0}\right)$ and damping $(\zeta)$; in GMMs, typically, $T_{0}$ ranges from 0.01 to $10 \mathrm{sec}$ and, $\zeta$ is equal to $5 \%$. The response of the oscillator depends on the frequency content and timing (compactness of energy) of the ground motion. From the entire frequency content of the ground motion, the response of the oscillator mainly depends on the amplitudes of the frequencies near and below $f_{0}$. Therefore, at small $T_{0}$ (high $f_{0}$ ), the response of the oscillator depends on the entire frequency content of the ground motion (i.e. spectral shape) and not just a narrow frequency bin. This makes the coefficients of a $P S A$ GMM at small $T_{0}$ magnitude dependent even for linear effects, as the shape of spectral acceleration response spectrum changes with magnitudes. The peak of a spectral acceleration response spectrum will be at $0.1 \mathrm{sec}$ for a magnitude $(M) 3$ event and at $0.3 \mathrm{sec}$ for a $M 7.5$ event (Figure $11)$; this means that at small magnitudes, the $P G A$ scaling (e.g. $V_{S 30}$ coefficient) will be consistent with the scaling of $T_{0}=0.1 \mathrm{sec}$, while at large magnitudes, the $P G A$ scaling will be consistent with $T=0.3 \mathrm{sec}$. This is also observed by Stafford et al. (2017), who showed that the linear site amplification factors are magnitude and distance dependent. A detailed discussion the differences between the scaling of $F A S$ and $P S A$ is given by Bora et al. (2016).

Most $P S A$ GMMs do not explicitly account for the magnitude dependence of the coefficients, such as the $V_{S 30}$ scaling or distance scaling; instead, they often use a limited range of magnitudes where the magnitude dependence of the coefficients is not pronounced. For instance, the data-set that was used in the development of the NGA West1 GMMs had a limited set of magnitudes that ranged from $M 4.5$ to $M 8$ Power et al. (2008). The approach of using a smaller range of magnitudes works when developing an ergodic GMM, as there is enough number of moderate-to-large magnitude events globally to estimate the coefficients, but it can be problematic when developing a non-ergodic GMM.

For the NGA West2 GMMs, the data set was extended to down to M3 with the objective of setting the reference ergodic model that could be used to evaluate regional differences in the site, path, and source terms based on small magnitude data. The NGA West2 GMMs modified the magnitude scaling to capture the average effect of the magnitude dependence of the coefficients, but this does not accurately model the magnitude dependence of the site and path effects. 


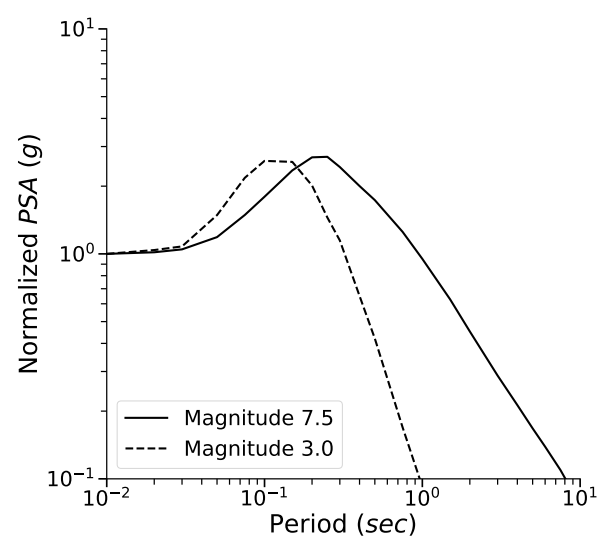

Fig. 1: Schematic of normalized response spectra for $M 3.0$ and 7.5 earthquakes

GMMs fall into two main categories: ergodic GMM and non-ergodic GMM. Ergodic GMMs assume that the statistical properties of a ground motion IM do not change in space (Anderson and Brune, 1999), and therefore, earthquakes and recordings from all around the world can be merged into a single dataset to estimate the GMM coefficients. Models developed under this assumption tend to have stable median estimates but large aleatory variability. Some models developed with the ergodic approach are: the NGA West GMMs for California Abrahamson et al. (2008), and the Douglas et al. (2014) GMM for Europe. Non-ergodic GMMs recognize that source, path, and site effects are systematically different at different parts of the world and account for these differences in the model development. Non-ergodic GMMs have smaller aleatory variability than ergodic GMMs, but in areas with sparse data, where the systematic effects are unknown, the reduced aleatory variability is accompanied by an increase in the epistemic uncertainty of the values of the median ground motion. The use of non-ergodic GMMs in Probabilistic Seismic Hazard Analysis (PSHA) is very promising, as the reduction in aleatory variability can have a large impact on the seismic hazard at large return periods, improving the accuracy of the site-specific hazard. A more in-depth discussion of ergodic and non-ergodic GMM is provided in the accompanying paper Lavrentiadis et al. (In press).

The estimation of the non-ergodic terms requires a large set of regional data. To achieve that, the datasets used in the development of non-ergodic GMM need to have a wider range of magnitudes to include the more frequent small-to-moderate earthquakes. It is this expansion of the magnitude range that makes the magnitude dependence of the GMM coefficients a more significant issue in non-ergodic GMMs. One solution to this problem is, first, develop a non-ergodic GMM for an $I M$ whose scaling does not suffer from the magnitude dependence, as $P S A$ does, and then for a scenario of interest, calculate the non-ergodic $P S A$ based on the non-ergodic $I M$ estimate.

The effective amplitude spectrum $(E A S)$, defined in Goulet et al. (2018), is one such $I M$ : the $E A S$ is a smoothed rotation-independent average power Fourier amplitude spectrum $(F A S)$ of the two horizontal components of an acceleration 
time history. In $E A S$, the amplitude at each frequency is independent of the amplitudes of the adjacent frequencies making the coefficients of an EAS GMM magnitude independent. Random vibration theory (RVT) provides a framework to calculate $P S A$ from $E A S$. It relies on extreme-value statistics to estimate the peak response of the oscillator directly in the Fourier domain; it does not require a phase-angle spectrum to first convert the ground motion in the time domain to compute the peak oscillator response. RVT has been used in the past to compute $P S A$ based on FAS from seismological theory (Hanks and McGuire, 1981; Boore, 1983, 2003) Other studies, such as Boore and Joyner (1984), Liu and Pezeshk (1999) Bora et al. (2015) and, Boore and Thompson (2012), focused on semi-empirical adjustments to the RVT framework to correct for the assumptions not satisfied by ground motions, mainly the fact that acceleration time histories are not stationary signals. More recently, Kottke et al. (In press) used RVT to develop an ergodic $P S A$ GMM for the eastern US based on an ergodic EAS GMM for the same region.

In this study, we developed two non-ergodic $P S A$ GMM. The average $P S A$ scaling is determined by backbone ergodic PSA GMMs. The non-ergodic effects are defined in terms of non-ergodic $P S A$ factors which are estimated by combining the Lavrentiadis et al. (In press) non-ergodic EAS GMM with RVT.

\section{Ground-Motion Data}

A subset of the NGAWest2 data-set (Ancheta et al., 2014) was used in this study. The selected subset contains the earthquake and stations that are located in California, western Nevada, and northern Mexico. Recordings that were flagged as questionable in Abrahamson et al. (2014) were removed from the regression subset Figure 2 shows the spatial distribution of earthquakes and stations. Most of the stations are located in Los Angeles, Bay Area, and San Diego metropolitan areas, whereas spatial density of the stations is lower in less populated areas, such as northern-eastern California. The regression data-set contains 7520 records from 185 earthquakes recorded at 1410 stations. Figure 3 shows the magnitude-distance distribution of the data and the number of records per frequency. The magnitude of the earthquakes ranges from 3.1 to 7.3 , and the distance of most records ranges from 10 to $200 \mathrm{~km}$. The usable frequency range of the majority of $E A S$ records spans from 0.4 and $20 \mathrm{~Hz}$. The minimum usable frequency of most $P S A$ records is $0.5 \mathrm{~Hz}$.

\section{Model development}

\subsection{Random-Vibration Theory}

RVT uses Parseval's theorem and extreme-value statistics ( $E V S)$ to estimate the $P S A$ based on the frequency content (i.e. $F A S$ ) and duration of a ground motion. Parseval's theorem is used to calculate the root-mean-square of the oscillator's response $\left(x_{r m s}\right)$ to the input ground motion, and a peak factor $(P F)$, based on $E V S$, is used to estimate the absolute peak response of the oscillator, which is the definition of PSA, based on $x_{r m s}$. PFs assume that the ground motion is a 


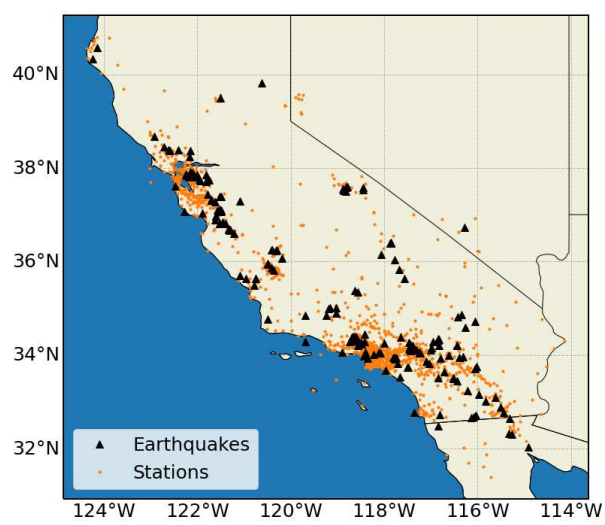

Fig. 2: Spatial distribution for earthquakes and station used in this study.

(a)

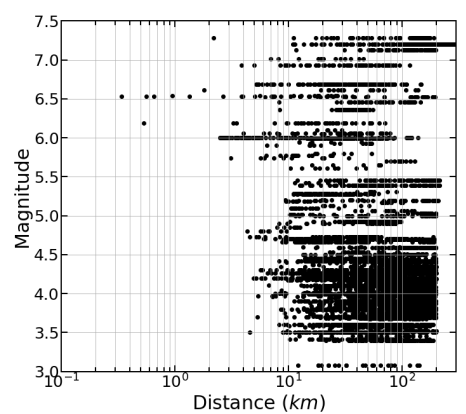

(b)



Fig. 3: Selected data from the NGAWest2 database. (a) Magnitude - Distance distribution, (b) number of $P S A$ and $E A S$ recordings per frequency used in the regression analysis

stationary stochastic process, and that it can be described as a band-limited white Gaussian noise with zero mean. The first assumption means that the amplitudes of the ground motion are identically distributed, and the second assumption means that the phase angles of the ground motion are randomly distributed. Although, earthquake ground motions violate both assumptions, numerous studies have shown that RVT provides PSA estimates that are in agreement with observed ground motions (Hanks and McGuire, 1981; Boore, 1983, 2003)

\subsubsection{Oscillator Response}

The response of an oscillator to a ground motion can be computed by convolving the ground motion with the impulse response $(I R)$ of the oscillator. The $I R$ is the 
(a)

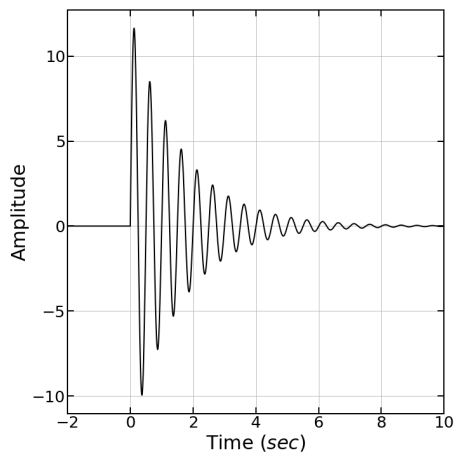

(b)

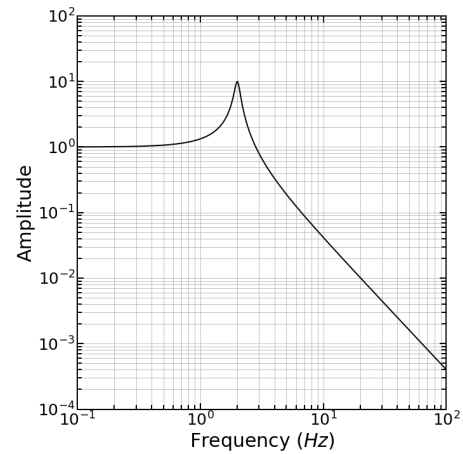

Fig. 4: Impulse response of a single degree of oscillator; (a) Time domain, (b) Fourier domain

response of an oscillator to a very brief acceleration pulse; that is a Dirac delta function. For an SDOF oscillator, the Fourier transform of the impulse response is:

$$
I R\left(f, f_{0}, \zeta\right)=\frac{-f_{0}^{2}}{f^{2}-f_{0}^{2}-2 j * \zeta * f_{0} * f}
$$

where, $f_{0}$ is the natural frequency of the oscillator, and $\zeta$ is the damping of the oscillator. As an example, Figure 4 shows the $P S A$ impulse response, in time and Fourier domain, for an SDOF oscillator with $f_{0}=2 \mathrm{~Hz}$ and $\zeta=5 \%$. In the Fourier domain, the convolution is performed by multiplying the ground motion's $F A S$ with $I R$; therefore, the response of an SDOF oscillator to a ground motion is:

$$
X(f)=F A S(f) I R_{S D}\left(f, f_{0}, \zeta\right)
$$

The $x_{r m s}$ of the oscillator's response is defined as:

$$
x_{r m s}=\sqrt{\frac{1}{D_{r m s}} \int_{-\infty}^{+\infty} x(t)^{2} d t}
$$

where $D_{r m s}$ is a measure of the duration which is defined in Section 3.1.4. Parseval's theorem states that the amount of energy in the time domain is equal to the amount of energy in the Fourier domain $\left(\int_{-\infty}^{+\infty} x(t)^{2} d t=2 \int_{0}^{+\infty} X(f)^{2} d f\right)$ which allows to compute $x_{r m s}$ directly in Fourier domain:

$$
x_{r m s}=\sqrt{\frac{1}{D_{r m s}} 2 \int_{0}^{+\infty} X(f)^{2} d f}=\sqrt{\frac{m_{0}}{D_{r m s}}}
$$

with $m_{0}$ being the zeroth moment of $F A S$. The $k^{\text {th }}$ moment of $F A S$ is defined as:

$$
m_{k}=2 \int_{0}^{+\infty}(2 \pi f)^{k} X(f)^{2} d f
$$




\subsubsection{Peak Factor}

The peak factor relates the $x_{r m s}$ with the maximum response of the oscillator $\left(x_{\max }\right)$, which is the definition of the $P S A$.

$$
P S A=P F x_{r m s}
$$

In general, $P F s$ fall into two main categories: those based on the Cartwright (1956) peak factor, abbreviated as CLH56, and those that are based on the Vanmarcke (1975) peak factor, abbreviated as V75.

In the first group, the CLH56 peak factor assumed that the peaks of a time history occur independently according to a Poisson process. In a series of papers, Boore and colleagues (Boore, 1983, Boore and Joyner, 1984, Boore, 2003) developed peak factors (BJ83) based on a reformulated version of CLH56 and removed an integrable singularity. Davenport (1964) proposed the a peak factor model (D64) based on an asymptotic form that approximates CLH56 for long time histories.

The main difference between V75 (Vanmarcke, 1975, 1976) and the PFs of the first group is that V75 dropped the Poisson process assumption. Because of this, V75 PF accounts for the time spend outside the threshold, which is important for a narrow-band process, and considers that the peaks could be clustered in time, which is important for a wide-band process. Der Kiureghian (1980) noted that the D64 peak factor overestimates the number of zero crossings, and developed a new $P F$ model (DK80) by modifying D65 PF so that it is asymptotically consistent with V75. V75 and D80 are in general agreement, but they deviate in time histories with a small number of zero crossings.

The V75 PF is selected for the development of the non-ergodic PSA GMM. V75 is preferred over the group of PF that are based on CLH56 due to the simplified assumptions in CLH56, and the complete form of V75 is preferred over the asymptotic forms, as the former is more accurate for the wide range of ground motions considered in this project. This choice is consistent with the $P F$ used in Kottke et al. (In press).

V75 expressed the probability distribution of the peaks as a first-passage problem. For a Gaussian process, the first-passage probability (i.e. the probability of no crossing) a $\pm a$ threshold (type-D barrier) in the time interval $(0, t)$ is equal to:

$$
P(|z|<r)=A \exp \left(-f_{z} t \exp \left(-r^{2} / 2\right) \frac{1-\exp \left(-\sqrt{\pi / 2} \delta_{e} r\right)}{1-\exp \left(-r^{2} / 2\right)}\right)
$$

where $r$ is the normalized barrier level $\left(r=a / x_{r m s}\right), A$ is the probability of starting within the thresholds $\left(A=1-\exp \left(-r^{2} / 2\right)\right), f_{z}$ is the average rate of zero crossings, and $\delta_{e}$ is an semi-empirical measure of bandwidth $\left(\delta_{e}=\delta^{1+b}\right) . b$ a non-negative constant which, in this case, is equal to 0.2 , and $\delta$ is a measure of bandwidth based on the spectral moments (Vanmarcke, 1972) defined as:

$$
\delta=\sqrt{1-\frac{m_{1}^{2}}{m_{0} m_{2}}}
$$

The cumulative distribution function $(\mathrm{CDF})$ of the peak values is obtained by setting $t$ equal to $D_{g m}$ in equation (7); that is, the probability of the peak of the 
time history being less than $r \times x_{r m s}$ is equal to the probability that the time history will remain within the thresholds $\pm r \times x_{r m s}$ for the entire ground-motion duration. With that, the CDF of $P F$ is equal to:

$$
\begin{aligned}
F_{P F}(r)= & \left(1-\exp \left(-r^{2} / 2\right)\right) \\
& \times \exp \left(-f_{z} D_{g m} \exp \left(-r^{2} / 2\right) \frac{1-\exp \left(-\sqrt{\pi / 2} \delta_{e} r\right)}{1-\exp \left(-r^{2} / 2\right)}\right)
\end{aligned}
$$

The expected value of $P F$ can be computed with the probability density function (PDF) of PF (Equation (10), which requires the derivation of the PDF. However, $P F$ is continuous and defined on the positive side of the real line; thus, the expected value of $P F$ can be computed directly from the CDF with equation Equation (11).

$$
\begin{gathered}
E[P F]=\int_{0}^{+\infty} r f_{P F}(r) d r \\
E[P F]=\int_{0}^{+\infty}\left(1-F_{P F}(r)\right) d r
\end{gathered}
$$

The mean estimate of the RVT $P S A$ can be computed by substituting the expected value of the $V 75 P F$ in Equation (6).

\subsubsection{Ground-Motion Duration}

In RVT, a measure of duration is needed in two steps: in the calculation of the peak factor, and in the calculation of $x_{r m s}$. Due to transient nature of a groundmotion, the duration measures used in these two steps are often different. $D_{g m}$ is the ground-motion duration, which is used in the calculation of PF; $D_{r m s}$ is the duration measure for the calculation of $x_{r m s}$. which is defined in section 3.1.4.

In seismology, the ground-motion duration is most commonly defined either as bracketed or as significant duration. Bracketed duration is the time interval between the first and last time the ground motion exceeds a threshold. Significant duration is the difference in time the normalized Arias intensity reaches two specific values. For instance, the $5-75 \%$ significant duration is the difference between the time the normalized Arias intensity is $5 \%$ and, the time the normalized Arias intensity is $75 \%$. The Arias intensity is defined as integral of the squared acceleration time history:

$$
I_{a}\left(t^{\prime}\right)=\frac{2 \pi}{g} \int_{0}^{t^{\prime}} x^{2}(t) d t
$$

The normalized Arias intensity, also known as Husid curve, is the ratio of $I_{a}$ at time $t$ over $I_{a}$ at the end of the ground motion:

$$
h(t)=\frac{I_{a}(t)}{I_{a}(+\infty)}
$$

In some RVT methods, $D_{g m}$ is set to a measure of significant duration, but in others, $D_{g m}$ is treated as a free parameter with units of time. For instance, Boore (2003) used the $D_{a 0.05-0.95}$ significant duration as $D_{g m}$, while Bora et al. (2015) 
and Bora et al. (2019) treated $D_{g m}$ as free parameter and developed a duration GMM with the goal to minimize misfit between the observed PSA and the PSA computed with RVT.

In this study, $D_{g m}$ is defined as an interval of significant duration. Different intervals of significant duration were tested as $D_{g m}$ candidates to find the one that minimized the misfit between the $P S A$ of the used dataset $\left(P S A_{N G A}\right)$ and the $P S A$ estimated with RVT ( $\left.P S A_{R V T}\right)$; the results of this comparison are shown in the Electronic supplement, Section S1. The $D_{a 0.05-0.85}$ significant duration resulted in the best fit of $P S A_{N G A}$ for the entire frequency range, 0.1 to $100 \mathrm{~Hz}$. The Abrahamson and Silva (1996) duration GMM (AS96) was selected for estimating $D_{a 0.05-0.85}$ for new scenarios, as to our knowledge, AS96 is the only GMM that provides an estimate for the selected duration interval. Despite the previous results, the $D_{a 5-75}, D_{a 5-95}, D_{v 5-75}$, and $D_{v 5-95}$ estimates of the Kempton and Stewart (2006) duration GMM and $D_{a 5-75}, D_{a 5-95}$, and $2 D_{a 20-80}$ estimates of the Afshari and Stewart (2016) duration GMM were evaluated as candidates for $D_{g m}$, but the $D_{a 0.05-0.85}$ of AS96 resulted to a better fit of $P S A_{N G A}$. The results of this comparison can be found in the Electronic supplement, Section S2.

The AS96 functional form for the mean estimate or the $D_{0.05-0.75}$ duration is:

$$
\ln D_{5-75}\left\{\begin{array}{lr}
\ln \left(\frac{1}{f_{c}}+c_{1}\left(R_{\text {rup }}-R_{c}\right)+c_{2} S\right) & \text { for } R_{\text {rup }} \geq R_{c} \\
\ln \left(\frac{1}{f_{c}}+c_{2} S\right) & \text { for } R_{\text {rup }}<R_{c}
\end{array}\right.
$$

where $f_{c}$ is the corner frequency of the earthquake:

$$
f_{c}=4.910^{6}\left(\frac{\Delta \sigma}{10^{1.5 M+16.05}}\right)
$$

$\beta$ is the shear-wave velocity at the source, and $\Delta \sigma$ is the stress drop. $1 / f_{c}$ is the source duration, $c_{1}\left(R_{\text {rup }}-R_{c}\right)$ captures the distance dependence, and $c_{2} S$ captures the site dependence. The scaling of AS96 has a physical basis because the distance and site dependence terms are additive, instead of multiplicative, to the source duration. The rational for an additive distance dependence is that small and large magnitude earthquakes are expected to have a similar increase of duration with increasing distance due to the scattering of the seismic waves. Similarly, the duration increase due to the site effects is also expected to be independent of the earthquake size. In AS96, other interval of significant duration can be calculated with Equation (16).

$$
\ln \left(\frac{D_{0.05-I}}{D_{5-75}}\right)=a_{1}+a_{2} \ln \left(\frac{I-0.05}{1-I}\right)+a_{3} \ln \left(\frac{I-0.05}{1-I}\right)^{2}
$$

\subsubsection{Correction for non-stationarity}

One of RVT's main assumptions that is violated when applied in ground motions is that the signal is stationary. Especially when predicting $P S A$ for large $T_{0}$, an SDOF oscillator will not abruptly stop at the end of the ground motion, instead it will have a transient decaying response, which if not considered, would lead to an overestimation of $x_{r m s}$. To solve this problem, Boore and Joyner (1984) (JB84) proposed to include the oscillator duration $\left(D_{o}\right)$ in $D_{r m s}$ as shown in Equation 
(17); $D_{o}$ is not included in the calculation of the $P F$ because the response of the oscillator follows a steady decay after the end of the excitation. Liu and Pezeshk (1999) (LP99) improved the estimate of $D_{o}$ by considering the spectral shape of the input time history in the $D_{o}$ scaling. Boore and Thompson (2012) (BT12), and Boore and Thompson (2015) (BT15) proposed a relationship for $D_{r m s} / D_{g m}$; they used a more flexible functional form compared to the previous studies and considered the magnitude and distance scaling of $D_{r m s} / D_{g m}$.

$$
D_{r m s}=D_{g m}+D_{o}
$$

The BT15 oscillator duration model was selected for the subsequent analyses, as in preliminary evaluations, the RVT PSA estimates with BT15 provided a better fit to the recorded $P S A$ than the alternative models. Although BT12 performed equally well in estimating the $P S A$ of medium-to-large earthquakes, it was not selected because its is not applicable to magnitudes less than 4 .

\subsubsection{Extrapolation of EAS}

To ensure that entire frequency content of the ground-motion is captured in the RVT calculations, both the ergodic and non-ergodic $E A S$ spectra are extrapolated at low and high frequencies. At low frequencies, $E A S$ is extrapolated to $0.01 \mathrm{~Hz}$ with an omega-square model (Brune, 1970):

$$
\begin{aligned}
& \Omega(f)=\frac{f^{2}}{1+f^{2} / f_{c}^{2}} \\
& E A S\left(f<f_{\text {min }}\right)=A_{f_{\text {min }}} \Omega(f)
\end{aligned}
$$

where $f_{c}$ is the corner frequency (Equation $(15)$ ), and $A_{f_{\min }}$ is the amplitude of the omega-squared model at the minimum frequency of the $E A S\left(f_{\min }\right)$. The stress drop for the calculation of $f_{c}$ for the omega-squared model is estimated with the Atkinson and Boore (2011) empirical relationship. $A_{f_{m i n}}$ is estimated based on the EAS amplitudes of $1.00 f_{\min }$ to $1.05 f_{\min }$ frequency bin:

$$
A_{f_{\text {min }}}=\operatorname{mean}\left(\frac{E A S(f)}{\Omega(f)}\right) \quad \text { for } f \in\left[1.0 f_{\min }, 1.05 f_{\min }\right]
$$

At high frequencies, $E A S$ is extrapolated to $100 \mathrm{~Hz}$ with a kappa model (Anderson and Hough, 1984):

$$
\begin{aligned}
& D(f)=\exp (-\pi \kappa f) \\
& E A S\left(f>f_{\max }\right)=A_{f_{\max }} D(f)
\end{aligned}
$$

$\kappa$ defines the rate of decay of the high frequencies, and $A_{f_{\max }}$ is the amplitude of the kappa model at the largest $E A S$ frequency, $f_{\max } . \kappa$ can be estimated with the Ktenidou et al. (2014) $\kappa-V_{S 30}$ empirical relationship:

$$
\ln (\kappa)=-0.4 \ln \left(\frac{V_{S 30}}{760}\right)-3.5
$$




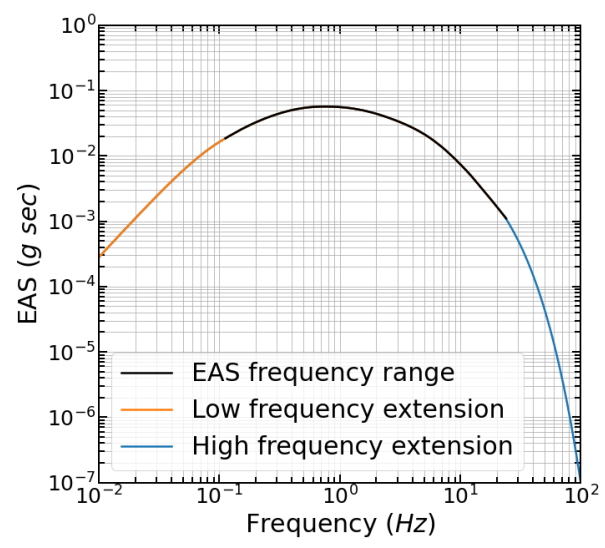

Fig. 5: Extrapolation of $E A S$ to low and high frequencies. $E A S$ is estimated for $M=7, R_{\text {rup }}=30 \mathrm{~km}$, and $V_{S 30}=400 \mathrm{~m} / \mathrm{sec}$.

$A_{f_{\max }}$ is estimated based on the $E A S$ amplitudes in the $0.95 f_{\max }$ to $1.00 f_{\max }$ frequency bin:

$$
A_{f_{\max }}=\operatorname{mean}\left(\frac{E A S(f)}{D(f)}\right) \quad \text { for } f \in\left[0.95 f_{\max }, 1.00 f_{\max }\right]
$$

As an example of the extrapolation procedure, the median estimate of the ergodic $E A S$ for a $M 7$ event, at a $R_{\text {rup }}$ distance of $30 \mathrm{~km}$, and a $V_{S 30}$ value of $400 \mathrm{~m} / \mathrm{sec}$ is extend to high and low frequencies using the omega-squared and kappa models in Figure 5 , which shows that the amplitudes of the extended frequencies are in agreement with the $E A S$ over the usable frequency range.

\subsubsection{RVT summary and validation}

In summary, all subsequent RVT calculations are performed with: the V75 PF, the median estimate of AS96 for $D_{a 0.05-0.85}$ as $D_{g m}$, BT15 for $D_{r m s}$, and the extrapolation procedure described in the previous subsection.

As a validation, Figure 6 shows the residuals between the natural-log of $P S A_{N G A}$ and the natural-log of $P S A_{R V T}$ with the recommended $R V T$ procedure. Overall, $P S A_{R V T}$ is in good agreement with $P S A_{N G A}$ for the entire period range $\left(T_{0}=0.01-10 \mathrm{sec}\right)$ with the fit improving for $M>5$. Figure 7 shows the mean and the standard deviation of the residuals versus $T_{0}$. The residuals have a positive bias at $T_{0}=1-4 \mathrm{sec}$; however, this is not propagated in the non-ergodic PSA GMM, as the GMM is developed using non-ergodic factors, which are defined in the next subsection (Section 3.2). The standard deviation or the residuals is approximately 0.2 natural-log units for the entire period range.

\subsection{Non-ergodic PSA factors}

The non-ergodic effects of the proposed $P S A$ GMM are expressed in terms of a non-ergodic $P S A$ factor $\left(F_{\text {nerg }}\right)$; that is, the difference of the logs the non-ergodic 
(a)

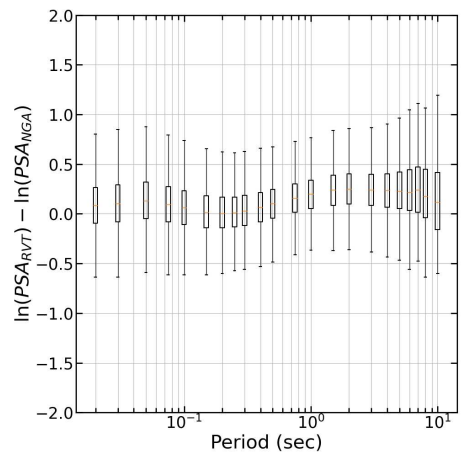

(b)

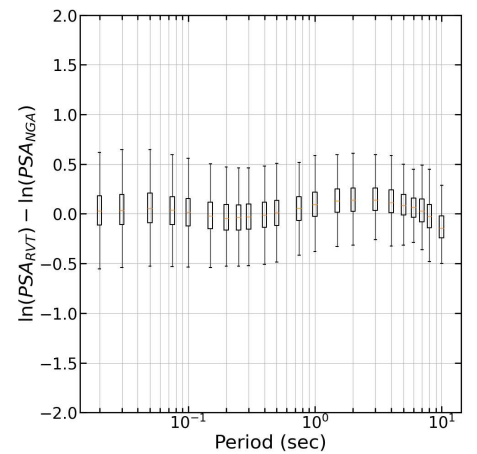

Fig. 6: Residuals between the records' $P S A$ and $P S A$ calculated with RVT. (a) residuals of records of all $M$, (b) residuals of records of $M>5$

(a)

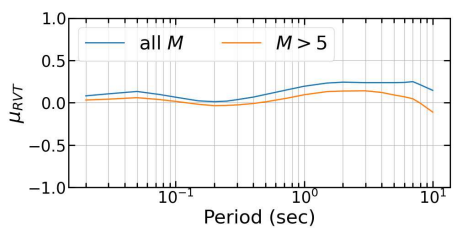

(b)

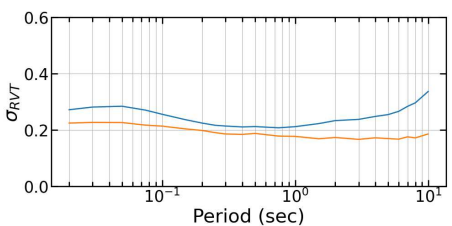

Fig. 7: Mean and standard deviation of the residuals between the records' $P S A$ and $P S A$ calculated with RVT.

$P S A$ estimate for a scenario of interest over the ergodic $P S A$ estimate for the same scenario (Equation (23)) The non-ergodic $P S A$ values are calculated with RVT and the Lavrentiadis et al. (In press) non-ergodic EAS GMM (LAK21), and the ergodic $P S A$ values are calculated with RVT and the Bayless and Abrahamson (2019) ergodic EAS GMM (BA18). The scenarios of interest are defined by the magnitude $(M)$, closest-rupture distance $\left(R_{\text {rup }}\right)$, time-average shear-wave velocity at the top $30 m\left(V_{S 30}\right)$, etc., which are input parameters to both the ergodic and non-ergodic EAS GMMs, but also the earthquake and site coordinates, $\vec{x}_{e}$ and $\vec{x}_{s}$, which define the source, path and site non-ergodic effects in LAK21. In this formulation, $F_{n e r g}$ captures the combined effect of all non-ergodic terms; there are no separate terms for the earthquake, path, and site non-ergodic effects.

$$
\begin{aligned}
F_{n e r g} & \left(T_{0}, M, R_{\text {rup }}, V_{S 30}, \vec{x}_{e}, \vec{x}_{s}, . .\right)= \\
= & \ln \left(P S A_{R V T}\left[\operatorname{IR}\left(T_{0}\right) E A S_{L A K 21}\left(M, R_{\text {rup }}, V_{S 30}, \vec{x}_{e}, \vec{x}_{s}, \ldots\right)\right]\right) \\
& -\ln \left(P S A_{R V T}\left[\operatorname{IR}\left(T_{0}\right) E A S_{B A 18}\left(M, R_{\text {rup }}, V_{S 30}, \ldots\right)\right]\right)
\end{aligned}
$$


The proposed non-ergodic PSA GMM is developed by coupling the aforementioned non-ergodic with an existing ergodic PSA GMM:

$$
\begin{aligned}
& y_{n e r g}\left(M, R, V_{S 30}, \vec{x}_{e}, \vec{x}_{s}, \ldots\right)= \\
& \quad=y_{\text {erg }}\left(M, R, V_{S 30}, \ldots\right)+F_{n e r g}\left(M, R, V_{S 30}, \vec{x}_{e}, \vec{x}_{s}, \ldots\right)
\end{aligned}
$$

where $y_{n e r g}$ is the natural log of the non-ergodic PSA median estimate, and $y_{\text {erg }}$ is the natural log of the ergodic median estimate. The benefit of this approach is that it separates the non-ergodic effects from the average ground-motion scaling. $F_{n e r g}$ does not affect the average scaling of the non-ergodic PSA GMM, as LAK21 is based on BA18, and thus, their average scaling is canceled out. Furthermore, the small bias of $R V T$ is also canceled out in this approach, as the same $R V T$ procedure is used to compute $P S A_{\text {erg }}$ and $P S A_{\text {nerg }}$ For the average scaling of the non-ergodic PSA GMM, yerg, we chose the Abrahamson et al. (2014) (ASK14) and Chiou and Youngs (2014) (CY14) ergodic PSA GMMs. Hereafter, the non-ergodic GMM that is based on ASK14 is called non-ergodic $\mathrm{GMM}_{1}$, and the non-ergodic GMM that is based on CY14 is called non-ergodic $\mathrm{GMM}_{2}$. The main reasons ASK14 and CY14 are selected to develop the non-ergodic GMM are: i) they were developed with the same data-set as BA18, and ii) they include complex scaling terms, such as hanging-wall effects, which can be passed to the non-ergodic GMMs.

The non-ergodic PSA GMM was not developed directly with RVT and LAK21 because this approach led to an overestimation the median PSA at medium-to-large periods. Figure 8 compares the four NGAWest2 GMMs: ASK14, BSSA14, CB14, and CY14 (Abrahamson et al. 2014; Boore et al., 2014, Campbell and Bozorgnia, 2014, Chiou and Youngs, 2014) with the spectral acceleration response spectrum created with RVT and BA18. The NGAWest2 GMMs are in good agreement with the $P S A$ from BA18 for the $M 5$ event, but the comparison worsens as the size of the earthquake increases. For periods $T_{0}=2-4 s e c$, for the $M 8$ earthquake, the PSA from BA18 is a factor of two higher than the NGAWest2 GMMs, indicating that, in this period range, BA18 has a stronger magnitude scaling than the NGAWest2 GMMs. Since LAK21 is based on BA18, a non-ergodic PSA GMM developed with RVT and LAK21 will also have a stronger magnitude scaling than the NGAWest2 GMMs. Due to the effort involved in the development of the NGAWest2 GMMs, we judge that their magnitude scaling is more likely to be correct, which is why we used the non-ergodic factors approach to develop the non-ergodic GMM; however, future studies should further investigate the cause of the different magnitude scaling.

The epistemic uncertainty of the non-ergodic PSA GMM is captured by sampling the non-ergodic terms of LAK21 GMM multiple times and calculating the $F_{n e r g}$ for each sample. As shown in the example in Section 4.1, it is important to consider the inter-frequency correlation of the non-ergodic terms, otherwise the epistemic uncertainty is underestimated.

\subsection{Constant Shift and Aleatory Model}

The constant shift $\left(\delta c_{0}\right)$, between-event residuals $\left(\delta B_{e}^{0}\right)$, and within-event withinsite residuals $\left(\delta W S_{e, s}^{0}\right)$ are estimated by fitting a mixed-effects linear model to the total residuals of the non-ergodic models:

$$
\epsilon_{e, s}=\delta c_{0}+\delta B_{e}^{0}+\delta W S_{e, s}^{0}
$$


(a)

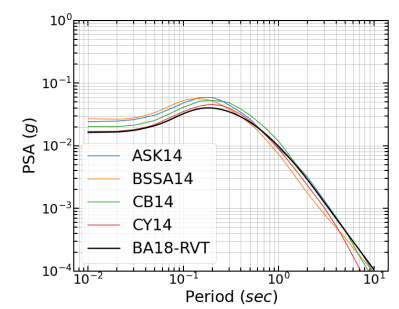

(b)

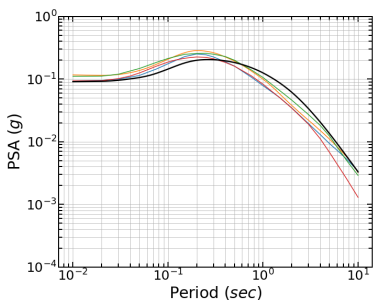

(c)

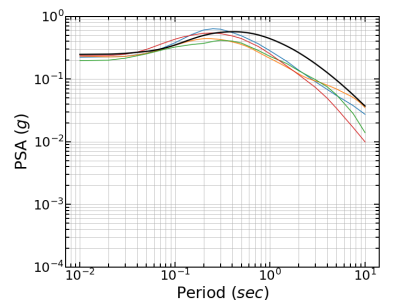

Fig. 8: Comparison of PSA spectra developed with the BA18 EAS GMM and RVT, shown with the black line, and $P S A$ spectra estimated using the NGAWest2 GMMs, shown with the colored lines. (a) $M 5.0$, (b) $M$ 6.5, and (c) $M 8.0$ earthquake scenario with $R_{\text {rup }}=30 \mathrm{~km}$ and $V_{S 30}=400 \mathrm{~m} / \mathrm{sec}$.

(a)



(c)



(b)

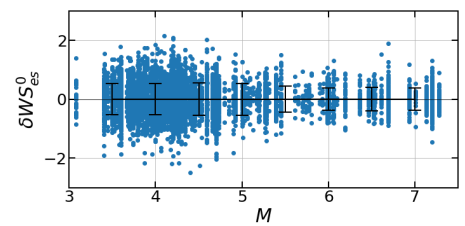

(d)



Fig. 9: Between-event and within-event within-site residuals for $T_{0}=0.25 \mathrm{sec}$ versus magnitude. (a) $\delta B_{e}$ of non-ergodic $\mathrm{GMM}_{1}$, (b) $\delta W S_{e, s}$ of non-ergodic $\mathrm{GMM}_{1}$, (c) $\delta B_{e}$ of non-ergodic $\mathrm{GMM}_{2}$, and (d) $\delta W S_{e, s}$ of non-ergodic $\mathrm{GMM}_{2}$.

The magnitude dependence of $\delta B_{e}^{0}$ and $\delta W S_{e, s}^{0}$ of the two non-ergodic $P S A$ GMMs for $T_{0}=0.25 \mathrm{sec}$ is evaluated in Figure 9. The mean of $\delta B_{e}^{0}$ and $\delta W S_{e, s}^{0}$ shows no trend with $M$, but their empirical standard deviation decreases with $M$. Similarly, the $R_{r u p}$ and $V_{S 30}$ dependence of the $\delta W S_{e, s}^{0}$ for $T_{0}=0.25 \mathrm{sec}$ is evaluated in Figures 10 and 11 where no significant trends are found in either the mean or the standard deviation.

Figure 12 shows the estimated and smoothed $\delta c_{0}$ of the two non-ergodic PSA GMMs. Non-ergodic $\mathrm{GMM}_{2}$, which is based on CY14, is only estimated up to $T_{o}=5 \mathrm{sec}$ because, at larger periods, $\delta c_{0}$ deviated significantly from zero.

Based on the empirical standard deviation of the non-ergodic residuals (Figure 9 ), both $\phi_{0}$ and $\tau_{0}$ are modeled as magnitude dependent (Equation (26) and (27)). Figure 13 shows the period dependence of $\phi_{0}$ and $\tau_{0}$ for small and large magnitudes. The magnitude dependence of $\phi_{0}$ and $\tau_{0}$ is more significant at small periods. The increase of the within-event aleatory variability at the small periods of small 
(a)

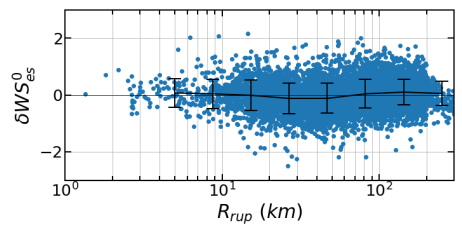

(b)

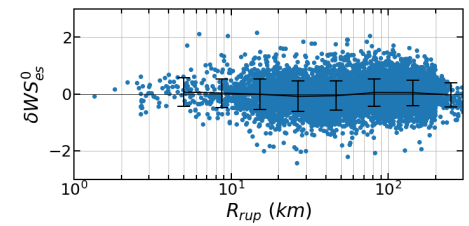

Fig. 10: Within-event within-site residuals for $T_{0}=0.25 \mathrm{sec}$ versus $R_{\text {rup }}$. (a) $\delta W S_{e, s}$ of non-ergodic $\mathrm{GMM}_{1}$, (b) $\delta W S_{e, s}$ of non-ergodic $\mathrm{GMM}_{2}$.

(a)

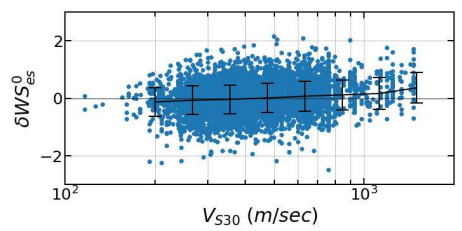

(b)

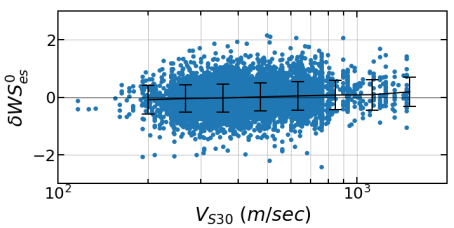

Fig. 11: Within-event within-site residuals for $T_{0}=0.25 \mathrm{sec}$ versus $V_{S 30}$. (a) $\delta W S_{e, s}$ of non-ergodic $\mathrm{GMM}_{1}$, (b) $\delta W S_{e, s}$ of non-ergodic $\mathrm{GMM}_{2}$.

(a)

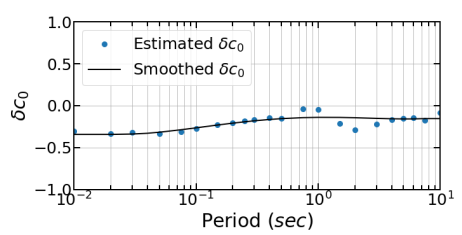

(b)



Fig. 12: Estimated and smoothed $\delta c_{0}$ versus $T_{0}$. (a) non-ergodic $\mathrm{GMM}_{1}$, (b) nonergodic $\mathrm{GMM}_{2}$

magnitudes may be caused by the radiation pattern which make the amplitude of the ground motion sensitive to the azimuthal angle. For large magnitudes, which can be thought as many small events, the radiation patterns have less impact on the ground-motion variability, because the individual radiation patterns destructively interfere with each other due to the different azimuthal angles. Similarly, the larger between-event aleatory variability at the small periods of small magnitudes is believed to be caused by differences in stress drop which shifts the ground motions at frequencies above the corner frequency of the earthquake. Due to the larger rupture dimensions of the large events, any variability in the stress drop along the rupture averages out resulting in reduced between-event variability. 
(a)

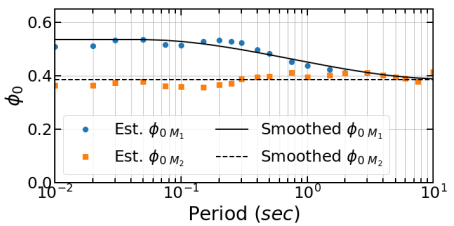

(c)

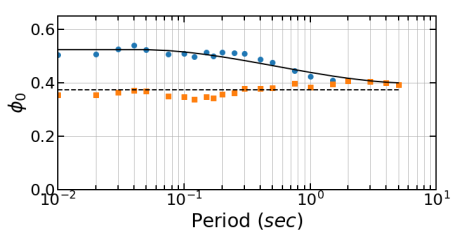

(b)

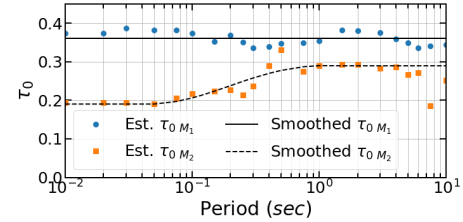

(d)

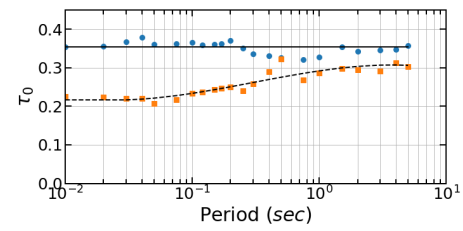

Fig. 13: Period dependence of aleatory model parameters. (a) period dependence of $\phi_{0 M_{1}}, \phi_{0 M_{1}}$ for non-ergodic $\mathrm{GMM}_{1}$ (b) period dependence of $\tau_{0 M_{1}}, \phi_{0 M_{1}}$ for non-ergodic $\mathrm{GMM}_{1}$ (c) period dependence of $\phi_{0 M_{1}}, \phi_{0 M_{1}}$ for non-ergodic $\mathrm{GMM}_{2}$ (d) period dependence of $\tau_{0 M_{1}}, \phi_{0 M_{1}}$ for non-ergodic $\mathrm{GMM}_{2}$

The total standard deviation of the two non-ergodic GMMs are 30 to $35 \%$ smaller than the total standard deviation of the ergodic GMMs.

$$
\begin{gathered}
\phi_{0}=\left\{\begin{array}{cl}
\phi_{0 M_{1}} & \text { for } M<5 \\
\phi_{0 M_{1}}+\left(\phi_{0 M_{2}}-\phi_{0 M_{2}}\right)(M-5) /(6.5-5) & \text { for } 5<M<6.5 \\
\phi_{0 M_{2}} & \text { for } M>6.5
\end{array}\right. \\
\tau_{0}=\left\{\begin{array}{cl}
\tau_{0 M_{1}} & \text { for } M<5 \\
\tau_{0 M_{1}}+\left(\tau_{0 M_{2}}-\tau_{0 M_{2}}\right)(M-5) /(6.5-5) & \text { for } 5<M<6.5 \\
\tau_{0 M_{2}} & \text { for } M>6.5
\end{array}\right.
\end{gathered}
$$

Figure 14 compares the proposed models for $\phi_{0}$ and $\tau_{0}$ with the standard deviations of the binned residuals for $T_{0}=0.25 \mathrm{sec}$. Overall, the aleatory models are in good agreement with the empirical standard deviations. The discrepancy at large magnitudes is considered acceptable, as the number of large magnitude events is small to reliably estimate the empirically standard deviation.

As a comparison with previous non-ergodic models, Figure 15 shows the total standard deviation of the two non-ergodic GMMs and the total standard deviation of the SWUS15 partially non-ergodic GMM (Abrahamson et al., 2015). The standard deviations of non-ergodic $\mathrm{GMM}_{1}$ and $\mathrm{GMM}_{2}$ are within the low and high branches of SWUS15 for entire period range for both small-to-moderate and large events. For small-to-moderate magnitude events and $T_{0}<1 \mathrm{sec}$, the total standard deviations of $\mathrm{GMM}_{1}$ and $\mathrm{GMM}_{2}$ are larger than the median branch of SWUS15. One possible reason for this is that $\sigma_{S S}$ of SWUS15 was estimated with magnitudes greater than 4 , whereas $\sigma_{0}$ of $\mathrm{GMM}_{1}$ and $\mathrm{GMM}_{2}$ were estimated with magnitudes greater than 3 which exhibit larger variability at small periods. At large events, the total standard deviations of $\mathrm{GMM}_{1}$ and $\mathrm{GMM}_{2}$ are between the central and lower branch of 
(a)

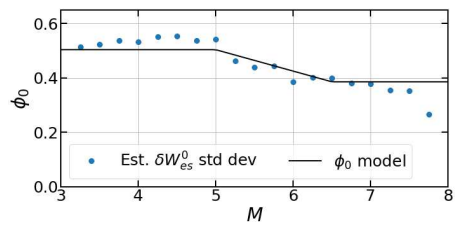

(c)

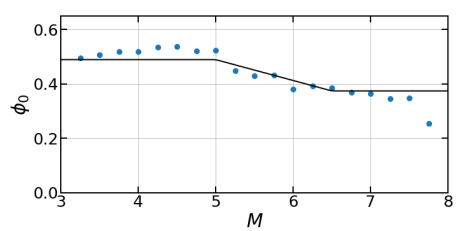

(b)

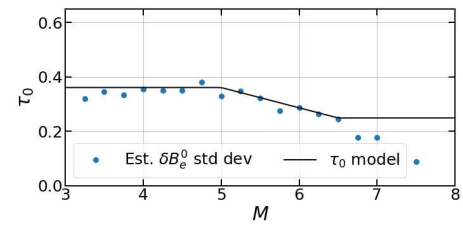

(d)

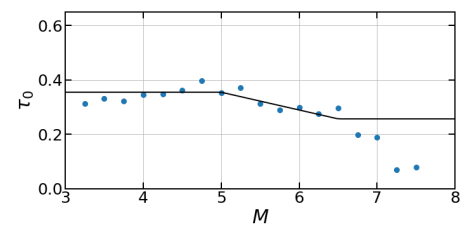

Fig. 14: Magnitude dependence of $\phi_{0}$ and $\tau_{0}$ for $T_{0}=0.25 \mathrm{sec}$. Circular markers denote the standard deviations of the binned residuals, and solid lines correspond to the standard deviation models. (a) $\phi_{0}$ for non-ergodic $\mathrm{GMM}_{1}$, (b) $\tau_{0}$ for non-ergodic $\mathrm{GMM}_{1}$, (a) $\phi_{0}$ for non-ergodic $\mathrm{GMM}_{2}$, and (b) $\tau_{0}$ for non-ergodic $\mathrm{GMM}_{2}$

(a)

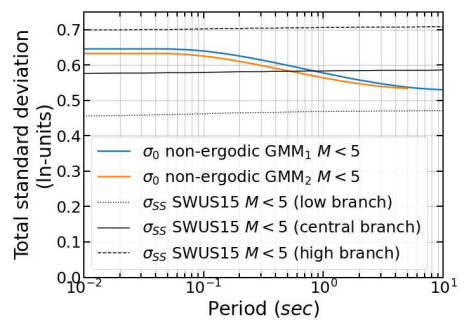

(b)

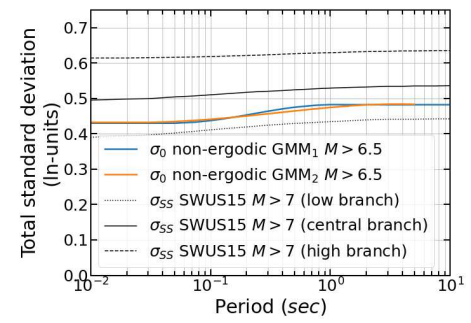

Fig. 15: Comparison of total standard deviation of non-ergodic $\mathrm{GMM}_{1}$ and $\mathrm{GMM}_{2}$ with total standard deviation of SWUS15 partially non-ergodic GMM. (a) smallto-moderate magnitude comparison, and (b) large magnitude comparison

SWUS15. The GMM 1 and $\mathrm{GMM}_{2} \sigma_{0}$ values are expected to be less than SWUS15 $\sigma_{S S}$ central branch because in addition to the systematic site effects, $\mathrm{GMM}_{1}$ and $\mathrm{GMM}_{2}$ capture the systematic source and path effects; however, the fact that the $\sigma_{0} \mathrm{GMM}_{1}$ and $\mathrm{GMM}_{2}$ are larger than the lower branch of SWUS15 means that the majority of the systematic effects captured by $\mathrm{GMM}_{1}$ and $\mathrm{GMM}_{2}$ are related to the site effects.

\section{Applications}

4.1 Effect of $E A S$ inter-frequency correlation in $F_{\text {nerg }} P S A$

In most GMMs, the ground-motion amplitude (i.e. $P S A$ or $E A S$ ) at every frequency is estimated independently; however, an actual ground-motion recording has peaks 
(a)

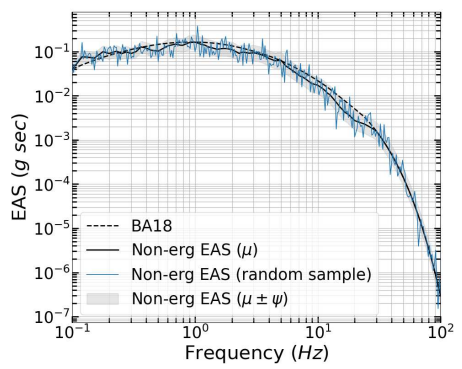

(b)

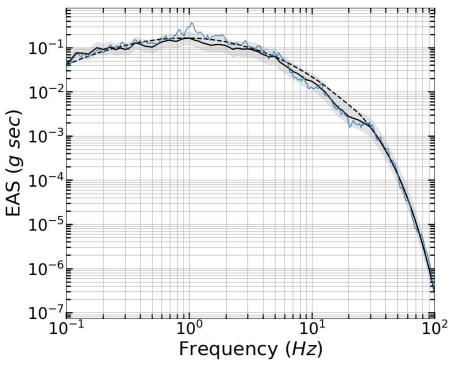

Fig. 16: Effective amplitude spectra for a $M 7$ earthquake in Hayward fault, $8 \mathrm{~km}$ away from a site located in Berkeley CA. (a) without inter-frequency correlation, and (b) with inter-frequency correlation.

and troughs. That is the amplitudes of neighbouring frequencies are correlated. For instance, if amplitude of some frequency is above the average, it is likely that amplitudes of the nearby frequencies will also be above the average. This inter-frequency correlation is important in RVT, as the response of an SDOF oscillator does not only depend on the ground-motion amplitude at $T_{0}$ but also at the frequency content around $T_{0}$. Bayless and Abrahamson (2018) showed that the $P S A$ variability is underestimated if the inter-frequency correlation of $F A S$ is not considered.

To illustrate the effect of the inter-frequency correlation in the calculation of $F_{n e r g} P S A$, we applied the proposed non-ergodic GMM with and without the inter-frequency correlation in $E A S$. In both cases, the scenario of interest is a $M 7$ earthquake in Hayward Fault $8 \mathrm{~km}$ away from a site in Berkeley, CA. The ergodic and non-ergodic $E A S$ of the two approaches are shown in Figure 16, and the corresponding non-ergodic $P S A$ spectra are shown in Figure 17. The non-ergodic $E A S$ in Figure 16 a are developed without inter-frequency correlation, whereas the non-ergodic $E A S$ in figure $16 \mathrm{~b}$ are developed using the inter-frequency correlation model in Lavrentiadis et al. (In press).

In $E A S$ space, both approaches resulted in the same median and epistemic uncertainty range, but in $P S A$ space, only the median is the same. The epistemic uncertainty of $P S A$ is larger when the $E A S$ inter-frequency correlation is considered, because if $E A S$ is at an extreme at $T_{0}$ it will generally stay at the extreme over the neighbouring frequencies; thus, all the frequencies which influence the response of the oscillator will constructively interfere leading to a range of $P S A$ amplitudes that is wider. In contrast, if the $E A S$ amplitudes are uncorrelated, they will have negating effect on the response of the oscillator, resulting in a narrower range of $P S A$. This shows the importance of considering the $E A S$ inter-frequency correlation in the non-ergodic $P S A$ calculations, as otherwise, the epistemic uncertainty of the $P S A$ is underestimated. 
(a)

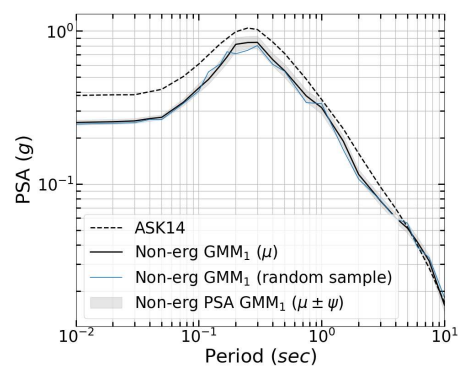

(b)

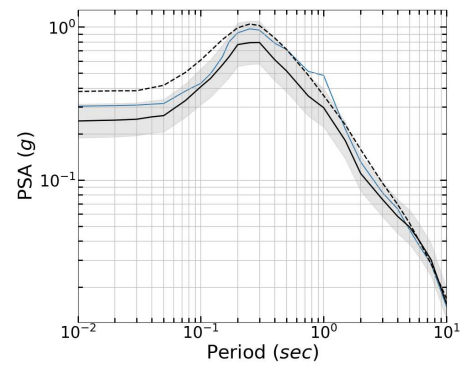

Fig. 17: $P S A$ spectra for a $M 7$ earthquake in Hayward fault, $8 \mathrm{~km}$ away from a site located in Berkeley CA. (a) without inter-frequency correlation, and (b) with inter-frequency correlation.

\subsection{Magnitude dependence $F_{n e r g} P S A$}

As an application example, Figures 18 and 19 present the $E A S$ and $P S A$ nonergodic for $T_{0}=0.1 \mathrm{sec}\left(f_{0}=10 \mathrm{~Hz}\right)$ for a $M 3$ and $M 8$ earthquake in San Andreas fault. The $E A S$ non-ergodic factors are magnitude independent; the median estimate and epistemic uncertainty of $F_{n e r g} E A S$ is the same in both events (Figure 18). The magnitude independence allows $F_{n e r g E A S}$ to be estimated from the more frequent small magnitude earthquakes and directly applied to the large magnitude events, which are typically of more interest. This is not the case for the PSA non-ergodic factors; $F_{n e r g P S A}$ depend on the spectral shape; which is why $F_{n e r g P S A}$ are different in the $M 3$ and $M 8$ earthquakes (Figure 19p, which illustrates why the non-ergodic PSA GMM is developed with non-ergodic factors that based on $E A S$. Most of the regional data that are used to estimate the non-ergodic effects are in form of small magnitude events, which couldn't be used if $P S A$ non-ergodic effects were estimated directly.

In addition, Figures 18 and 19 show the spatial distribution of the epistemic uncertainty. In this example, where the location of the earthquake is fixed, the spatial distribution of the epistemic uncertainty depends on the path and site location. Both the $E A S$ and $P S A$ epistemic uncertainties are small near stations that have recorded past events, whereas in remote areas with no available groundmotion data to constrain the non-ergodic terms, the epistemic uncertainties are larger.

The evaluation of the magnitude dependence of the $E A S$ and $P S A$ non-ergodic factors is further examined in Figures 20 and 21. The three scenarios in this comparison are a $M 3,5.5$ and 8 event in San Andreas Fault, $105 \mathrm{~km}$ from the site in San Francisco, CA. As mentioned previously, the non-ergodic $E A S$ factors are the same for all three events (Figure 20), while the non-ergodic $P S A$ factors are different, especially at small periods (Figure 21), $T_{0}<0.1 \mathrm{sec}$. This happens because, for $f_{0}>10 \mathrm{~Hz}\left(T_{0}<0.1 \mathrm{sec}\right)$, there is little ground-motion content in $E A S$ to resonate the SDOF oscillator, making its response, and subsequently $P S A$, depended on the peak of each spectrum. Similarly, the non-ergodic PSA factors for $T_{0}<0.1 \mathrm{sec}$ depend on the non-ergodic $E A S$ factors at the peak of each spectrum. In this example, the $M 3$ event has the largest non-ergodic $P S A$ factors 
(a)

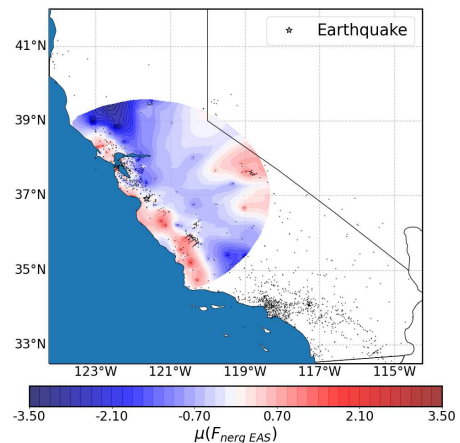

(c)

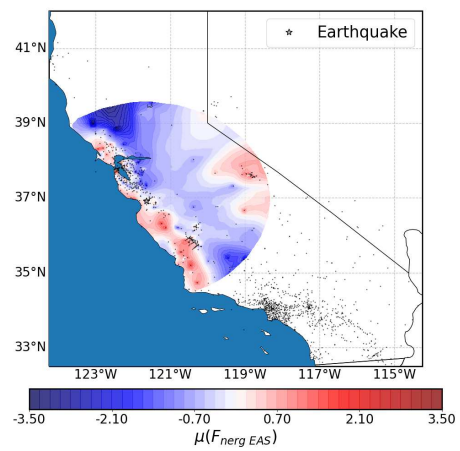

(b)

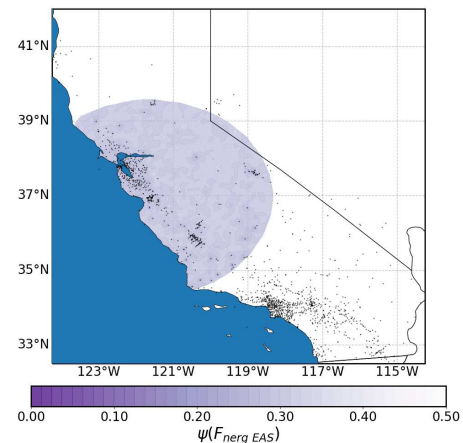

(d)

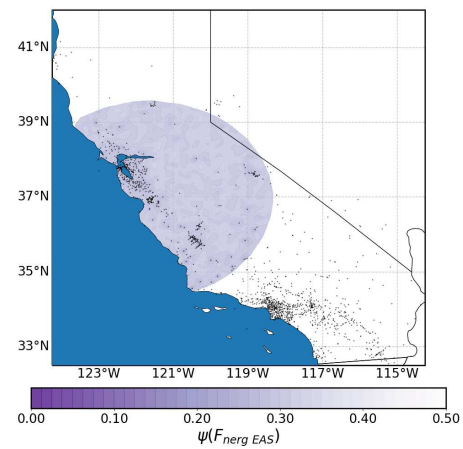

Fig. 18: $E A S$ non-ergodic factors, $F_{n e r g} E A S$, for $f_{0}=10 H z$ for an earthquake in San Andreas. The star corresponds to the earthquake location, and the dots correspond the location of the stations in the used dataset. (a) mean of $F_{\text {nerg }} E A S$ for $M=3.0$, (b) epistemic uncertainty of $F_{n e r g} E A S$ for $M=3.0$ (c) mean of $F_{n e r g} E A S$ for $M=8.0$, and (d) epistemic uncertainty of $F_{n e r g} E A S$ for $M=8.0$

at $T_{0}<0.1 \mathrm{sec}$, because the non-ergodic $E A S$ factors are predominately positive over its peak $(f=2$ to $6 H z)$. The $M 8$ event has the smallest non-ergodic $P S A$ factors at $T_{0}<0.1 \mathrm{sec}$ because its peak $(f<0.1$ to $6 \mathrm{~Hz})$ encompasses the dip of the non-ergodic $E A S$ factors that occur from $f=0.3$ to $2 \mathrm{~Hz}$.

\subsection{Example Hazard Calculations}

A comparison of the ergodic and non-ergodic PSHA results for $P S A\left(T_{0}=0.25 \mathrm{sec}\right)$ for a site in Berkeley, CA is presented in Figure 22. The PG\&E source model was used in all hazard calculations (Pacific Gas and Electric Company (PG\&E), 2015, 2017). The ergodic hazard calculations were performed with the ASK14 and CY14 GMMs, with equal weights, while the non-ergodic hazard calculations were performed with non-ergodic $\mathrm{GMM}_{1}$ and $\mathrm{GMM}_{2}$, with equal weights. The epistemic uncertainty of the non-ergodic GMMs was captured by 100 realizations 
(a)



(c)

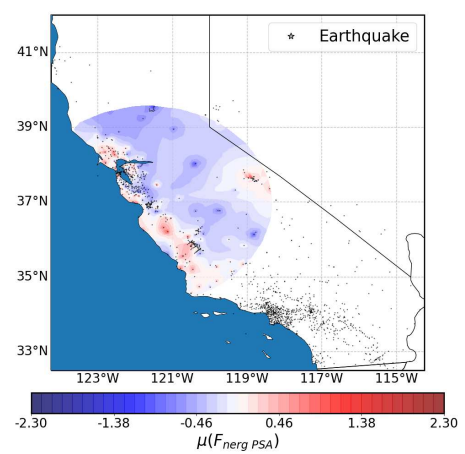

(b)

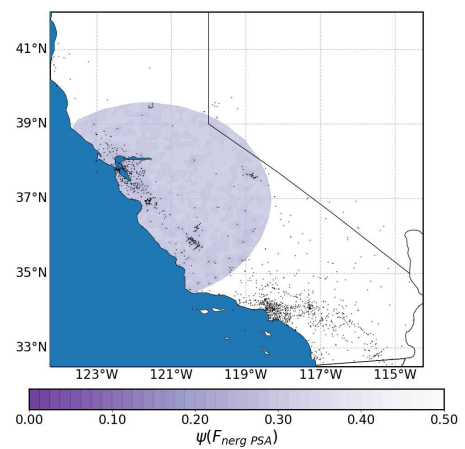

(d)

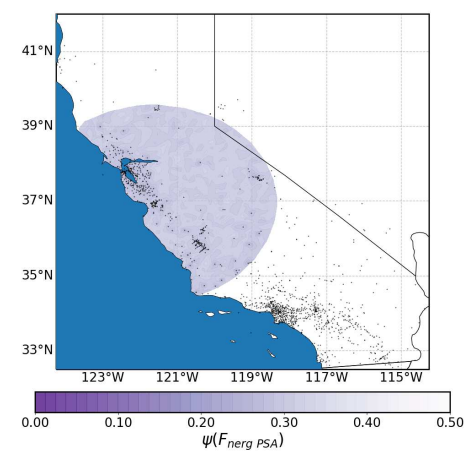

Fig. 19: $P S A$ non-ergodic factors, $F_{n e r g} P S A$, for $T_{0}=0.1 s e c$ for an earthquake in San Andreas. The star corresponds to the earthquake location, and the dots correspond the location of the stations in the used dataset. (a) mean of $F_{n e r g} P S A$ for $M=3.0$, (b) epistemic uncertainty of $F_{n e r g} P S A$ for $M=3.0$ (c) mean of $F_{n e r g} P S A$ for $M=8.0$, and (d) epistemic uncertainty of $F_{n e r g} P S A$ for $M=8.0$

(a)

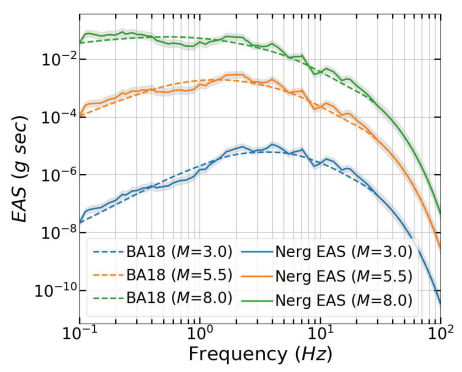

(b)



Fig. 20: (a) Ergodic and non-ergodic $E A S$ for $M 3,5.5$, and 8 earthquakes in San Andreas fault, $105 \mathrm{~km}$ from a site in San Francisco, CA (b) non-ergodic $E A S$ factors for the same scenarios. 
(a)

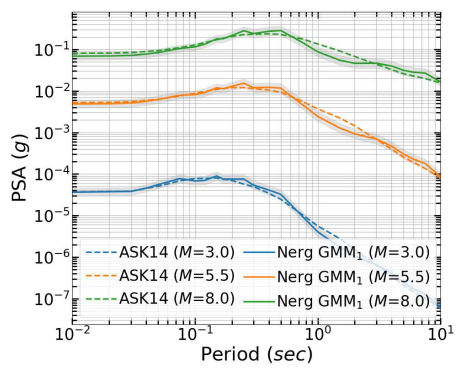

(b)



Fig. 21: (a) Ergodic and non-ergodic PSA spectra for $M 3,5.5$, and 8 earthquakes in San Andreas fault, $105 \mathrm{~km}$ from a site in San Francisco, CA (b) non-ergodic $P S A$ factors for the same scenarios.

of $F_{\text {nerg }} P S A$. This leads to a logic tree with 200 branches; each branch is a combination of a non-ergodic model $\left(\mathrm{GMM}_{1}\right.$ or $\left.\mathrm{GMM}_{2}\right)$ and a $F_{n e r g}$ PSA sample.

The difference between the two non-ergodic hazard calculations is that, in Figure 22b, only the regional systematic site-effects are constrained, while, in Figure 22c recordings from past earthquakes are assumed to be available and thus, both the regional and site-specific site effects are constrained. The regional site effects are captured by the $\delta c_{1 a, s}$ term of LAK21 GMM which is a function of the site location. The site-specific site effects are captured by the $\delta c_{1 b, s}$ term of LAK21 GMM, which can be determined either from past recordings or through a site-specific site response analysis.

For the ergodic hazard calculations, the mean hazard curve is flater than the non-ergodic hazard curves due to the large aleatory variability of ASK14 and CY14, and the epistemic uncertainty is small as it only encompasses the epistemic uncertainty in the seismic source characterization and the median scaling of the ground motion averaged over all of California. Comparing the two non-ergodic calculations, the mean hazard curve is flatter and the epistemic uncertainty is larger in Figure $22 \mathrm{~b}$ as $\delta c_{1 b, s}$ is free. This example shows the impact of non-ergodic GMM in PSHA where at moderate-to-large return periods it can lead to a factor of two to four change in the mean ground-motion level.

\section{Conclusions}

A new approach to develop non-ergodic PSA GMMs is presented in this study which considers the magnitude dependence of the non-ergodic terms. Due to the linear properties of Fourier Transform, a non-ergodic EAS GMM is used to estimate the non-ergodic effects from the small magnitude events and transfer them to the events of interest. RVT is used to compute the non-ergodic $P S A$ effects based on the non-ergodic $E A S$ effects, while the average scaling of the non-ergodic $P S A$ GMM is controlled by an existing ergodic PSA GMM.

Two non-ergodic PSA GMMs are developed in this study. The first one uses the ASK14 GMM as a backbone model for the average scaling and is applicable to periods $T_{0}=0.01-10 \mathrm{sec}$. The second one uses the CY14 GMM as a backbone 
(a)

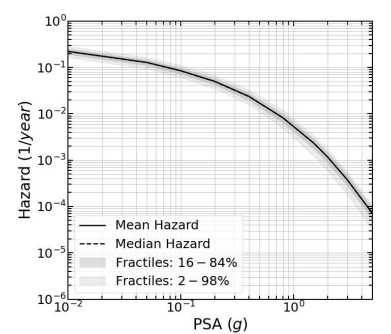

(b)

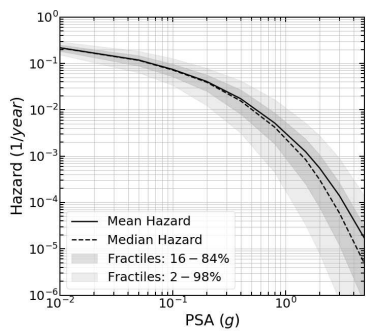

(c)

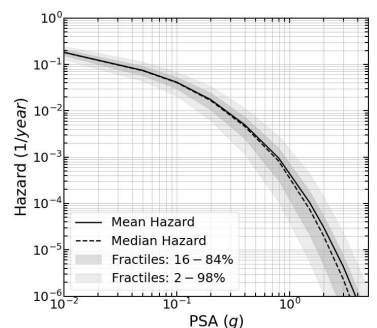

Fig. 22: Hazard results: mean, median, and 2-98\%, $16-84 \%$ fractile ranges of total hazard at $T=0.25 \mathrm{sec}$ for a site in Berkeley, CA. (a) Ergodic hazard calculation, (b) Non-ergodic hazard with unconstrained zero-correlation site term $\left(\delta c_{1 b, s}\right)$ of LAK21 GMM, (c) Non-ergodic Hazard with constrained zero-correlation site term $\left(\delta c_{1 b, s}\right)$ of LAK21 GMM.

model for the average scaling and is applicable to periods $T_{0}=0.01-5 \mathrm{sec}$. The non-ergodic $P S A$ effects are quantified in terms of non-erodic $P S A$ factors, that is the difference between the log of $P S A$ estimated with RVT and the non-ergodic $E A S$ and the log of $P S A$ estimated with RVT and the ergodic EAS. In both cases, the LAK21 GMM is used for the non-ergodic $E A S$ and the BA18 GMM is used for the ergodic $E A S$. The RVT calculations are performed with the V75 PF, the median estimate of $D_{a 5-85}$ from AS96 for the ground-motion duration, and the BT15 for the oscillator duration. The RVT components were chosen based on a thorough evaluation of alternative models for the peak factors, ground-motion duration and oscillator duration. The objective of the evaluation was to minimize misfit between the observed $P S A$ and the $P S A$ computed with RVT.

The advantages of developing the non-ergodic GMM with an ergodic backbone model and non-ergodic $P S A$ factors, instead of developing it directly with RVT and the LAK21 are: i) the elimination of the small bias of $R V T$ at $T_{0}=1-4 \mathrm{sec}$, ii) the separation of the non-ergodic effects from average scaling, and iii) the adoption of complex scaling terms present in ergodic PSA GMMs. Compared to the recorded $P S A$, the $P S A$ estimated with RVT has a small positive bias at $T_{0}=1-4 \mathrm{sec}$. This bias is not propagated in the non-ergodic $P S A$ factors; it is canceled out, as both the ergodic and non-ergodic RVT PSA estimates are calculated with the same approach.

Aleatory aleatory variability of the two non-ergodic $P S A$ GMMs is approximately 30 to $35 \%$ smaller than the aleatory variability of an ergodic PSA GMM.

Future studies should reevaluate the RVT and EAS models so that when combined they result in a $P S A$ predictions consistent with $P S A$ GMMs. Furthermore, the proposed non-ergodic GMMs were developed with a subset of the NGAWest2 database which was compiled in 2014. As larger data sets which include more recent and more frequent small magnitude events become available, the proposed models should be assessed and potentially expanded with additional non-ergodic terms. Similarly, $3 D$ broadband numerical simulations or inferred intensity measurements from historical earthquakes should be used to evaluate the efficacy of the proposed models. 
${ }_{543}$ Non applicable

${ }_{545}$ Code availability

\section{Declarations}

Funding Seismic Program.

Conflict of interest

Ethics approval

Non applicable

Non applicable

owledgements This work was partially supported by the PG\&E Geosciences Department Long-Term Seismic Program. The authors thank Nicolas Kuehn for instructive comments on an early draft of this manuscript.

This work was partially funded by the PG\&E Geosciences Department Long-Term

The authors declare that they have no conflict of interest.

Consent to participate

Consent for publication

Availability of data and material

${ }_{546}$ The are python scripts for the non-ergodic regressions are provided at:

547 https://github.com/glavrentiadis/NonErgodicGMM_public 


\section{References}

Abrahamson NA (2021) Haz45.3. https://github.com/abrahamson/HAZ

Abrahamson NA, Silva WJ (1996) Apendix A: Empirical Ground Motion Models, Description and Validation of the Stochastic Ground Motion Model. Tech. rep., Brookhaven National Laboratory, New York, NY

Abrahamson NA, Atkinson GM, Boore DM, Bozorgnia Y, Campbell KW, Chiou BS, Idriss IM, Silva WJ, Youngs RR (2008) Comparisons of the NGA ground-motion relations. Earthquake Spectra 24(1):45-66, DOI 10.1193/1.2924363

Abrahamson NA, Silva WJ, Kamai R (2014) Summary of the ASK14 ground motion relation for active crustal regions. Earthquake Spectra 30(3):1025-1055, DOI 10.1193/070913EQS198M

Abrahamson NA, Al-Atik L, Bayless J, Dinsick A, Dreger DS, Gregor N, Kuehn N, Walling M, Watson-Lamprey J, Wooddell K, Youngs RR (2015) Southwestern united states ground motion characterization sshac level 3. Tech. rep., GeoPentech, rev. 2

Afshari K, Stewart JP (2016) Physically Parameterized Prediction Equations for Significant Duration in Active Crustal Regions. Earthquake Spectra 32(4):20572081, DOI 10.1193/063015EQS106M, URL http://journals . sagepub.com/doi/ 10.1193/063015EQS106M

Ancheta TD, Darragh RB, Stewart JP, Seyhan E, Silva WJ, Chiou BS, Wooddell KE, Graves RW, Kottke AR, Boore DM, Kishida T, Donahue JL (2014) NGA-West2 database. Earthquake Spectra 30(3):989-1005, DOI 10.1193/070913EQS197M

Anderson JG, Brune JN (1999) Probabilistic seismic hazard analysis without the ergodic assumption. Seismological Research Letters 70(1):19-28, DOI 10.1785/ gssrl.70.1.19

Anderson JG, Hough SE (1984) Spectrum of Acceleration At High Frequencies. Bulletin of the Seismological Society of America 74(5):1969-1993

Atkinson GM, Boore DM (2011) Modifications to existing ground-motion prediction equations in light of new data. Bulletin of the Seismological Society of America 101(3):1121-1135, DOI 10.1785/0120100270

Bates D, Mächler M, Bolker B, Walker S (2015) Fitting linear mixed-effects models using lme4. Journal of Statistical Software 67(1):1-48, DOI 10.18637/jss.v067.i01

Bayless J, Abrahamson NA (2018) Evaluation of the interperiod correlation of ground-motion simulations. Bulletin of the Seismological Society of America 108(6):3413-3430, DOI 10.1785/0120180095

Bayless J, Abrahamson NA (2019) Summary of the BA18 Ground-Motion Model for Fourier Amplitude Spectra for Crustal Earthquakes in California. Bulletin of the Seismological Society of America 109(5):2088-2105, DOI 10.1785/0120190077,

URL https://pubs.geoscienceworld.org/ssa/bssa/article/109/5/2088/ 573508/Summary-of-the-BA18-GroundMotion-Model-for-Fourier

Boore DM (1983) Stochastic simulation of high-frequency ground motions based on seismological models of the radiated spectra. Bulletin of the Seismological

Society of America 73(6):1865-1894, URL http://www.bssaonline.org/cgi/ content/abstract/73/6A/1865

Boore DM (2003) Phase Derivatives and Simulation of Strong Ground Motions. Bulletin of the Seismological Society of America 93(3):1132-1143, DOI 10.1785/

0120020196, URL https://pubs.geoscienceworld.org/bssa/article/93/3/ $1132-1143 / 147015$ 
Boore DM, Joyner WB (1984) A note on the use of random vibration theory to predict peak amplitudes of transient signals. Bulletin of the Seismological Society of America 74(5):2035-2039, URL http://www.bssaonline.org/content/74/ 5/2035.short

Boore DM, Thompson EM (2012) Empirical improvements for Estimating earthquake response spectra with random-vibration theory. Bulletin of the Seismological Society of America 102(2):761-772, DOI 10.1785/0120110244

Boore DM, Thompson EM (2015) Revisions to some parameters used in stochasticmethod simulations of ground motion. Bulletin of the Seismological Society of America 105(2):1029-1041, DOI 10.1785/0120140281

Boore DM, Stewart JP, Seyhan E, Atkinson GM (2014) NGA-West2 equations for predicting PGA, PGV, and 5\% damped PSA for shallow crustal earthquakes. Earthquake Spectra 30(3):1057-1085, DOI 10.1193/070113EQS184M

Bora SS, Scherbaum F, Kuehn NM, Stafford PJ, Edwards B (2015) Development of a response spectral ground-motion prediction equation (GMPE) for seismichazard analysis from empirical fourier spectral and duration models. Bulletin of the Seismological Society of America 105(4):2192-2218, DOI 10.1785/0120140297

Bora SS, Scherbaum F, Kuehn N, Stafford P (2016) On the relationship between fourier and response spectra:Implications for the adjustment of empirical groundmotion prediction equations (GMPEs). Bulletin of the Seismological Society of America 106(3):1235-1253, DOI 10.1785/0120150129

Bora SS, Cotton F, Scherbaum F (2019) NGA-West2 empirical fourier and duration models to generate adjustable response spectra. Earthquake Spectra 55(1):61-93, DOI 10.1193/110317EQS228M

Brune JN (1970) Tectonic stress and the spectra of seismic shear waves from earthquakes. Journal of Geophysical Research 75(26):4997-5009, DOI 10.1029/ jb075i026p04997

Campbell KW, Bozorgnia Y (2014) NGA-West2 ground motion model for the average horizontal components of PGA, PGV, and 5\% damped linear acceleration response spectra. Earthquake Spectra 30(3):1087-1114, DOI 10.1193/062913EQS175M

Cartwright DE (1956) The statistical distribution of the maxima of a random function. Proceedings of the Royal Society of London Series A Mathematical and Physical Sciences 237(1209):212-232, DOI 10.1098/rspa.1956.0173

Chiou BS, Youngs RR (2014) Update of the Chiou and Youngs NGA Model for the Average Horizontal Component of Peak Ground Motion and Response Spectra. Earthquake Spectra 30(3):1117-1153, DOI 10.1193/072813EQS219M, URL http://peer.berkeley.edu/lifelines/nga_docs/jul_10_06/Chiou_Youngs_ NGA_2006.pdfhttp://earthquakespectra.org/doi/10.1193/072813EQS219M

Davenport AG (1964) Note on the Distribution of the Largest Value of a Random Function With Application To Gust Loading. Proceedings of the Institution of Civil Engineers 28(2):187-196, DOI 10.1680/iicep.1964.10112

Der Kiureghian A (1980) Structural response to stationary excitation. Journal of the Engineering Mechanics Division 106(6):1195-1213

Douglas J, Akkar S, Ameri G, Bard PY, Bindi D, Bommer JJ, Bora SS, Cotton F, Derras B, Hermkes M, Kuehn NM, Luzi L, Massa M, Pacor F, Riggelsen C, Sandıkkaya MA, Scherbaum F, Stafford PJ, Traversa P (2014) Comparisons among the five ground-motion models developed using RESORCE for the prediction of response spectral accelerations due to earthquakes in Europe 
and the Middle East. Bulletin of Earthquake Engineering 12(1):341-358, DOI $10.1007 /$ s10518-013-9522-8

Goulet C, Kottke A, Boore D, Bozorgnia Y, Hollenback J, Kishida T, Der Kiureghian A, Ktenidou O, Kuehn N, Rathje E, et al. (2018) Effective amplitude spectrum (eas) as a metric for ground motion modeling using fourier amplitudes. In: 2018 Seismology of the Americas Meeting

Hanks TC, McGuire RK (1981) The character of high-frequency strong ground motion. Bull Seism Soc Amer 71:1897-1919

Kempton JJ, Stewart JP (2006) Prediction equations for significant duration of earthquake ground motions considering site and near-source effects. Earthquake Spectra 22(4):985-1013, DOI 10.1193/1.2358175

Kottke A (2020) arkottke/pyrvt v0.7.2. Zenodo, DOI 10.5281/zenodo.3630729, URL https://doi.org/10.5281/zenodo.3630729

Kottke AR, Abrahamson NA, Boore DM, Bozorgnia Y, Goulet C, Hollenback J, Kishida T, Der Kiureghian A, Ktenidou OJ, Kuehn NM, Rathje EM, Silva WJ, Thompson EM, Wang X (In press) Selection of Random Vibration Theory Procedures for the NGA East Project and Ground Motion Modeling. Earthquake Spectra (1):1-16, DOI 10.1177/XXXX

Ktenidou OJ, Cotton F, Abrahamson NA, Anderson JG (2014) Taxonomy of $\kappa:$ A review of definitions and estimation approaches targeted to applications. Seismological Research Letters 85(1):135-146, DOI 10.1785/0220130027

Lavrentiadis G, Abrahamson NN, Kuehn NM (In press) A non-ergodic effective amplitude ground-motion model for california. Bulletin of Earthquake Engineering

Liu L, Pezeshk S (1999) An improvement on the estimation of pseudoresponse spectral velocity using RVT method. Bulletin of the Seismological Society of America 89(5):1384-1389

Pacific Gas and Electric Company (PG\&E) (2015) Seismic source characterization for the diablo canyon power plant, san luis obispo county, california. Tech. rep., Pacific Gas and Electric Company (PG\&E), San Francisco, CA

Pacific Gas and Electric Company (PG\&E) (2017) Deterministic seismic hazard results report: Pg\&e system-wide hydro risk project. Tech. rep., Pacific Gas and Electric Company (PG\&E), San Francisco, CA

Power M, Chiou BS, Abrahamson NA, Bozorgnia Y, Shantz T, Roblee C (2008) An overview of the NGA project. Earthquake Spectra 24(1):3-21, DOI 10.1193/ 1.2894833

R Core Team (2020) R: A Language and Environment for Statistical Computing. R Foundation for Statistical Computing, Vienna, Austria, URL https://www R-project.org/

Stafford PJ, Rodriguez-Marek A, Edwards B, Kruiver PP, Bommer JJ (2017) Scenario dependence of linear site-effect factors for short-period response spectral ordinates. Bulletin of the Seismological Society of America 107(6):2859-2872, DOI 10.1785/0120170084

Van Rossum G, Drake FL (2009) Python 3 Reference Manual. CreateSpace, Scotts Valley, CA

Vanmarcke EH (1972) Properties of spectral moments with applications to random vibration. ASCE Journal of the Engineering Mechanics Division 98(EM2):425-446

Vanmarcke EH (1975) On the Distribution of the First-Passage Time for Normal Stationary Random Processes. Journal of Ap- 
694 plied Mechanics 42(1):215-220, DOI 10.1115/1.3423521, URL https:

695 //asmedigitalcollection.asme.org/appliedmechanics/article/42/1/215/

$696387707 / 0 n-t h e-D i s t r i b u t i o n-o f-t h e-F i r s t P a s s a g e-T i m e-f o r$

${ }_{697}$ Vanmarcke EH (1976) Structural Response to Earthquakes. In: Develop-

${ }_{698}$ ments in Geotechnical Engineering, Elsevier Inc., pp 287-337, DOI

699 10.1016/B978-0-444-41494-6.50011-4, URL http://dx.doi.org/10.1016/

700 B978-0-444-41494-6.50011-4https://linkinghub.elsevier.com/retrieve/

701 pii/B9780444414946500114 


\section{Supplementary Files}

This is a list of supplementary files associated with this preprint. Click to download.

- electronicsupplement.pdf 